\title{
MEDICAL IMAGE SEGMENTATION FOR ANATOMICAL KNOWLEDGE EXTRACTION
}

\author{
${ }^{1}$ Ms Maya Eapen and ${ }^{2}$ Reeba Korah \\ ${ }^{1}$ Faculty of Computer Science and Engineering, Jerusalem College of Engineering, Chennai, India \\ ${ }^{2}$ Faculty of Electronics and Communication Engineering, St Joseph's College of Engineering, Chennai, India
}

Received 2014-01-11; Received 2014-01-17; Accepted 2014-02-22

\begin{abstract}
Computed Tomography-Angiography (CTA) images of the abdomen, followed by precise segmentation and subsequent computation of shape based features of liver play an important role in hepatic surgery, patient/donor diagnosis during liver transplantation and at various treatment stages. Nevertheless, the issues like intensity similarity and Partial Volume Effect (PVE) between the neighboring organs; left the task of liver segmentation critical. The accurate segmentation of liver helps the surgeons to perfectly classify the patients based on their liver anatomy which in turn helps them in the treatment decision phase. In this study, we propose an effective Advanced Region Growing (ARG) algorithm for segmentation of liver from CTA images. The performance of the proposed technique was tested with several CTA images acquired across a wide range of patients. The proposed ARG algorithm identifies the liver regions on the images based on the statistical features (intensity distribution) and orientation value. The proposed technique addressed the aforementioned issues and been evaluated both quantitatively and qualitatively. For quantitative analysis proposed method was compared with manual segmentation (gold standard). The method was also compared with standard region growing.
\end{abstract}

Keywords: CTA Image, Region Growing, Liver Segmentation, Image Classification, Shape-Based Features

\section{INTRODUCTION}

In the recent years, the population of patients approaching for the treatment of liver related diseases and surgeries are increasing rapidly. Abdominal CT scan has emerged as a painless, noninvasive and reliable medical test for patient diagnosis and treatment. Preferably the pathological condition of liver is assessed with its shape based features. In today's world, vast amount of image data are generated in each field, such as medical image (CT, MRI, PET), satellite images and all kinds of digital photographs. It is difficult for the users to discover useful and implicit information from these images. Image mining is a process that can automatically discover this implicit information and patterns from this high volume of Images (Ashraf et al., 2012). Image mining is more than just an extension of data mining to image domain; it can be viewed as an interdisciplinary endeavor that draws upon computer vision, image processing, image Corresponding Author: Ms Maya Eapen, Faculty of Computer Science and Engineering, Jerusalem College of Engineering, Chennai, India

retrieval, machine learning, artificial intelligence, database and data mining, (Ashraf et al., 2012).

The information/knowledge that can be mined from medical images can be classified as (Xiang et al., 2012): anatomical, physiological and pathological. Anatomical information pertains to the location and extent of organ structures, while physiological data deals with the function of the organ and finally the Pathological information refers to the abnormalities related to or caused by diseases that manifest in the medical images. Even though the radiologists are trained for Anatomical localization of organs, it is a tedious and time-consuming task.

However, automatic and precise delineation or segmentation of organs can enable organ-specific quantitative analysis and improve the reporting process (Ashraf et al., 2012; Kumar and Kumar, 2014). From the context of the proposed work, we acquire anatomical information of liver through the segmentation of liver from abdominal CTA images. The anatomical information mainly includes the computation of various 
shape based features of liver and labeling of organ of interest. In addition, the segmentation of liver is the first and foremost step for acquiring anatomical and pathological information like liver cancer, identification of liver sections.

In the literature, many models have been proposed for liver segmentation from abdominal CT/MRI images with varying degree of success (Chen et al., 2012). (Campadelli et al., 2009) proposed a labeling algorithm; organs are labeled based on the intensity values and spatial relationship between the neighboring voxels. The biggest volume corresponding to the lowest label is the searched rough liver boundary. The over-and under-segmentation is handled with histogram information. (Luo and Seneviratne, 2012) fused the statistical with the structural features to achieve a better segmentation. These features are extracted at the pixel level and a trained SVM was used to classify image into liver pixel or non-liver pixels.

In the literature, many models have been proposed for liver segmentation from abdominal CT/MRI images with varying degree of success (Chen et al., 2012). (Campadelli et al., 2009) proposed a labeling algorithm; organs are labeled based on the intensity values and spatial relationship between the neighboring voxels. The biggest volume corresponding to the lowest label is the searched rough liver boundary. The over-and undersegmentation is handled with histogram information. (Luo and Seneviratne, 2012) fused the statistical with the structural features to achieve a better segmentation. These features are extracted at the pixel level and a trained SVM was used to classify image into liver pixel or non-liver pixels.

Segmentation based on shape based models (Daisy and Selvi, 2014). (Badakhshannoory and Saeedi, 2011) focus on the liver shape and they fall short of accuracy in segmentation due to liver-shape variability among various patients. The popular Statistical Shape Model (SSM) (Heimann and Meinzer, 2009), is extended to liver segmentation in (Zhang et al., 2010), using a 3D Generalized Hough Transform (GHT) method to detect approximate location of the liver shape model. After the SSM adaption, the shape model is deformed to adapt to the liver contour through an optimal surface detection based on graph theory. The over segmentation problem of traditional watershed algorithm (Masoumi and Pourmina, 2012) is mitigated by adjusting the parameters of the algorithm iteratively with the aid MLP neural networks. The neural networks are trained to estimate the shape based features. Foruzan et al. (2009), authors estimate an initial boundary of the liver based on the anatomical knowledge of the liver and its surrounding tissues furthermore, a heuristic based technique is utilized to segment the liver region from the slices. Achieving a perfect training data that accommodates the variant liver shapes among patients is a major difficulty encountered in the shape based liver segmentation algorithms.

The major challenges hidden in the segmentation of liver from abdominal CTA images arise from the following fact; the intensity similarity of liver with its adjacent organs such as heart and kidney (Lim, 2009). It makes it difficult to delineate the organs by considering the intensity discontinuity alone due to the PVE (blurred boundaries) at the regions where the organs touch each other. The proposed system combines the intensity with the orientation feature to address the aforementioned challenges. The study is organized as follows: In section 2 we present the methods employed in the proposed liver segmentation system. Section 3 illustrates the experimental results and performance evaluation of the proposed method. Finally, we conclude the study in section 4.

\section{PROPOSED SYSTEM}

The Segmentation phase of the proposed technique for liver segmentation is depicted in Fig. 1. The proposed technique consists of following phases

- Image Acquisition and pre-processing of abdominal CTA image namely:

- Advanced Region growing for liver segmentation

- Anatomical knowledge Extraction from the segmented liver

- Performance evaluation of the proposed system

\subsection{Image Acquisition and Preprocessing}

The input to the proposed system is an abdominal 2D CTA DICOM slices, with a resolution of $512 \times 512$ pixels. Majority of the CT/MRI images are noisy and suffer from blurred edges. In abdominal images, it is even more difficult to identify boundary of structures due to the PVE at the regions where the organs touch each other. Hence, the preprocessing step focus to enhance the edges and reduce the noise level in the input images before dealing with segmentation process. This module includes Image resizing, histogram equalization, ROI selection (Image cropping) and median filtering. In our method, a global Histogram equalization (Yousuf and Rakib, 2011; Karthikeyan and Rengarajan, 2014) is used which is a perfect technique for contrast and texture enhancement of medical images. Based on the anatomical knowledge, as much as unnecessary information should be removed from the images in the preprocessing stage. 


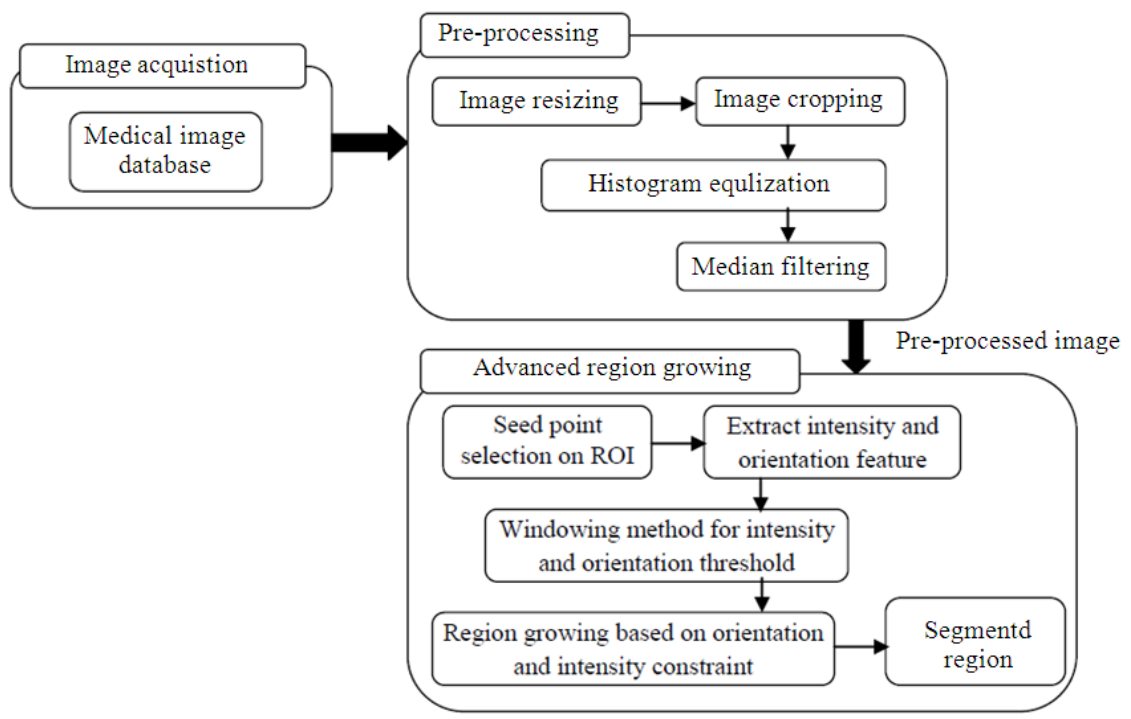

Fig. 1. Block diagram of proposed segmentation technique

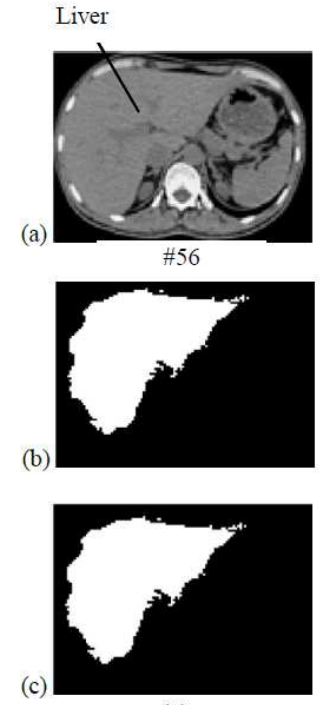

(1)
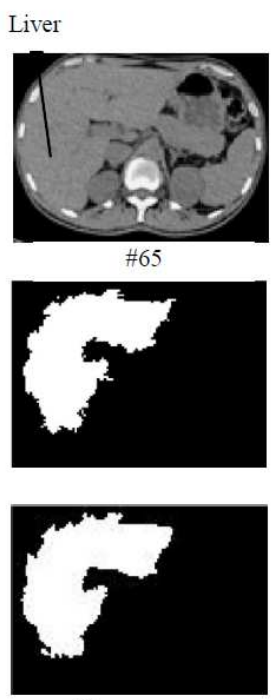

(2)

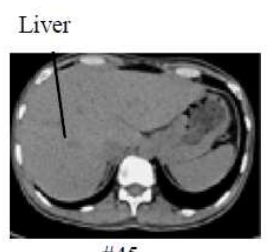

\#45
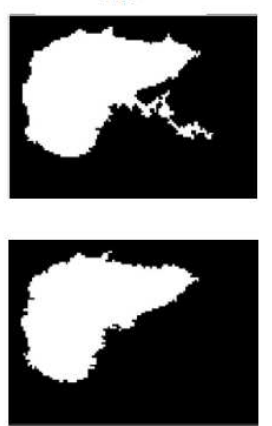

(3)
Liver Heart
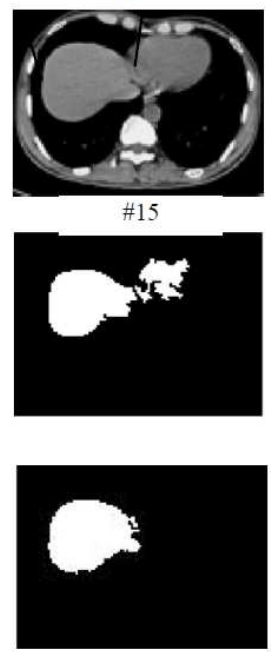

(4)

Fig. 2. Result comprison (a) preprocessed original images (b) RG (c) proposed method

This deletion process reduces the ROI size significantly and thereby reduces the computation complexity and increases the performance of the segmentation algorithm. Median filtering is an edge enhancement technique (Karthikeyan and Rengarajan, 2014). All these three sequential steps are performed for all the given images. The preprocessing stage makes region boundaries clearer and removes small noisy regions. Examples of certain preprocessed 2D CTA images are shown in Fig. 2.

\subsection{Advanced Region Growing Technique}

Region growing is a thresholding based technique. The technique involves the selection of an initial seed point in the Region of Interest (ROI) on the given CTA preprocessed image I. In our case ROI is the liver region, so a seed point is selected almost near the cntre of the liver region. From the manually or automatically selected seed point, RG approach examines the neighboring pixels of the seed point and determines their inclusion in the ROI based on certain constraints. Due to intensity 
similarity of liver with adjacent organs, traditional region growing method based on intensity constraint alone can result in over-segmentation or holes (Foruzan et al., 2009). Therefore, in our Advanced Region Growing (ARG) technique we have integrated gradient orientation constraint with the intensity constraint in the region growing process.

In ARG, two thresholds are considered namely intensity and gradient orientation thresholds represented as IntT and GOT respectively. As the region grows from manually or automatically selected seed point $\mathrm{p}$ on $\mathrm{I}$, pixels that satisfy both the intensity threshold $\left(\mathrm{Int}_{\mathrm{T}}\right)$ and orientation threshold $\left(\mathrm{GO}_{\mathrm{T}}\right)$, are added as part of the liver region. Threshold values denote the maximum values by which neighboring pixels can differ from the pixel of interest (seed point).The newly added region pixels becomes the new seed points.

The orientation of each pixel is determined from the gradient of the input CTA image. The image gradient makes the edges of the objects more distinguishable. Moreover, the gradient direction or orientation adds smooth constraint to the boundaries (Chen et al., 2012). Let the $\mathrm{I}_{\mathrm{x}}$ and $\mathrm{I}_{\mathrm{y}}$ be the gradients in the $\mathrm{x}$ and $\mathrm{y}$ directions respectively for the give image I. The gradient values are represented in radians. Subsequently, the gradient values in the $\mathrm{x}$ and $\mathrm{y}$ direction are combined as in equation (1) to determine the orientation of each pixel Equation (1):

$$
G(i, j)=\frac{1}{1+\left(I x^{2}+I^{2}\right)}
$$

The resultant $\mathrm{G}$ is a gradient matrix with orientation value of pixel at location $\mathrm{i}$ and $\mathrm{j}$. In the proposed advanced region growing method, the neighboring pixel is added to the region if it satisfies the constraints defined as Equation (2):

$$
\left|\mathrm{I}_{\mathrm{p}}-\mathrm{I}_{\mathrm{n}}\right| \leq \mathrm{Int}_{\mathrm{T}} \text { and }\left|\mathrm{G}_{\mathrm{p}}-\mathrm{G}_{\mathrm{n}}\right| \leq{\mathrm{G} 0_{\mathrm{T}}}
$$

where, $I_{p}$ and $G_{p}$ are the intensity and orientation of the pixel of interest and $I_{n}$ and $G_{n}$ are the neighbor pixels. The threshold values int ${ }_{\mathrm{T}}$ and $\mathrm{GO}_{\mathrm{T}}$ are calculated according to Equation 3 and 4 respectively in a window of size $9 \times 9$ centered for initial seed point pixel p Equation (3):

$$
\operatorname{Int}_{\mathrm{T}}=\left|\mathrm{I}_{\mathrm{p}}-\frac{1}{\mathrm{~N}} \times \sum_{\mathrm{i}-4}^{\mathrm{i}+4} \sum_{\mathrm{j}-4}^{\mathrm{j}+4} \mathrm{I}_{\mathrm{ij}}\right|
$$

$$
\mathrm{G}_{\mathrm{T}}=\left|\mathrm{G}_{\mathrm{p}}-\frac{1}{\mathrm{~N}} \times \sum_{\mathrm{i}-4}^{\mathrm{i}+4} \sum_{\mathrm{j}-4}^{\mathrm{j}+4} \mathrm{G}_{\mathrm{ij}}\right|
$$

where, $\mathrm{N}$ is the total number of pixels in the window, here its 81 and $\mathrm{I}_{\mathrm{ij}}$ and $\mathrm{G}_{\mathrm{ij}}$ are the intensity and orientation value at pixel location $i$ and $j$.

Algorithm: Advanced Region Growing

Input: Pre-processed Image, I

Output: Segmented Liver region Is, with liver pixels and non-liver pixels marked as 1 and 0 respectively

1. Calculate the gradient of $\mathrm{I}$ in the $\mathrm{x}$ and $\mathrm{y}$ direction as $\mathrm{I}_{\mathrm{x}}$ and $\mathrm{I}_{\mathrm{y}}$

2. Combine $I_{x}$ and $I_{y}$ according to Equation (1) to generate gradient matrix $\mathrm{G}$.

3. Compute the Intensity threshold ( $\left(\mathrm{Int}_{\mathrm{T}}\right)$ and orientation threshold $\left(\mathrm{GO}_{\mathrm{T}}\right)$ as given in Equation (3) and (4).

4. For the neighboring pixel of selected seed point $S_{\mathrm{P}}$, check for the constraints as in Equation (2).

5. If both the constraints are satisfied, neighboring pixels are added to the liver region and neighboring pixels forms the new seed point.

6. Repeat steps 5 until all pixels in I are labeled as liver $(=1)$ or non-liver pixels $(=0)$ to form the segmented binary image $\mathrm{I}_{\mathrm{s}}$

\subsection{Anatomical Knowledge Extraction}

The segmented liver region can be further analyzed to extract the anatomical information. The various anatomical shape based information of the liver includes: Perimeterarea ratio, orientation and minor to major axis ratio. The computation of shape based features is detailed in section 3 . This anatomical information helps the radiologist to categorize or classify the patients for further analysis. Furthermore, it helps in describing salient objects in an image and therefore assists in image retrieval.

\section{EXPERIMENTS AND RESULTS}

The proposed method has been evaluated on the datasets obtained from the reputed institutions. It is implemented with MATLAB 7.9 on a Windows XP System. The liver regions were identified and manually segmented by expert and trained radiologists. The manually segmented images served as the reference standard (gold standard). The performance of the proposed method is validated both quantitatively and qualitatively. 


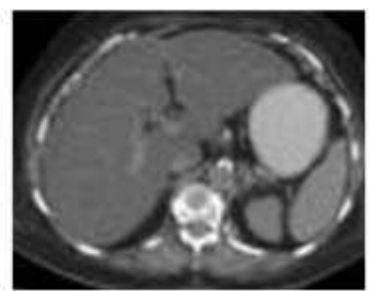

(a)

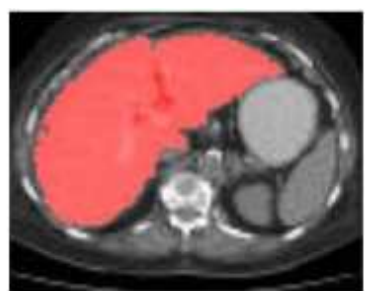

(b)
Fig. 3. (a) CTA slice (b) segmented liver region in red color

Table 1. The calculated features of liver extracted from Fig. 2 using manual method

\begin{tabular}{llll}
\hline & Calculated features & \\
Slice no. & Orientation (O) & Min/Maj (AR) & Per/Area (PA) \\
\hline$\# 56$ & 37.30 & 0.43 & 0.38 \\
$\# 65$ & 37.98 & 0.47 & 0.39 \\
$\# 45$ & 40.94 & 0.40 & 0.41 \\
$\# 15$ & 41.98 & 0.51 & 0.23 \\
\hline
\end{tabular}

Table 2. The calculated features of liver extracted from images in Fig. 1 using proposed method

Calculated features

\begin{tabular}{lllll}
$\begin{array}{l}\text { Slice } \\
\text { No. }\end{array}$ & $\begin{array}{l}\text { Orientation } \\
(\mathrm{O})\end{array}$ & $\begin{array}{l}\text { Min/Maj } \\
(\mathrm{AR})\end{array}$ & $\begin{array}{l}\text { Per/Area } \\
(\mathrm{PA})\end{array}$ & $\begin{array}{l}\text { Error } \\
\text { term }(\mathrm{E})\end{array}$ \\
\hline$\# 56$ & 37.20 & 0.44 & 0.36 & $7.76 \times 10^{-10}$ \\
$\# 65$ & 38.03 & 0.43 & 0.40 & $5.86 \times 10^{-11}$ \\
$\# 45$ & 39.84 & 0.43 & 0.40 & $1.01 \times 10^{-07}$ \\
$\# 15$ & 41.62 & 0.46 & 0.20 & $2.36 \times 10^{-07}$ \\
\hline
\end{tabular}

The quantitative analysis (Chen et al., 2012; Masoumi and Pourmina, 2012) is conducted based on shape based features including the Orientation (O), Perimeter to Area Ratio (PA) and minor to major Axis Ratio (AR) of the segmented liver region. Table 1 and 2 depict values of the shape based features for the manually segmented and with the proposed method respectively for images given in Fig. 2. Table 2 depicts error term (Chen et al., 2012), E which is as follows Equation (5):

$$
\mathrm{E}=\left(\mathrm{O}-\mathrm{O}_{\mathrm{r}}\right)^{2}\left(\mathrm{PA}-\mathrm{PA}_{\mathrm{r}}\right)^{2}\left(\mathrm{AR}-\mathrm{AR}_{\mathrm{r}}\right)^{2}
$$

where, O,PA and $\mathrm{AR}$ are the features extracted from manually segmented reference images, $\mathrm{O}_{\mathrm{r}}, \mathrm{PA}_{\mathrm{r}}$ and $\mathrm{AR}_{\mathrm{r}}$ are extracted from images segmented using proposed method. The negligible Error term (E) shows that the proposed method is efficient in liver region extraction.
For qualitative analysis, the proposed method is compared with traditional Region Growing (RG) algorithm. Since only the intensity constraint is considered for region growing, an over segmentation can be observed on slices where the liver shares its boundary with heart. However, due to intensity similarity of heart and liver, RG fails to delineate liver-heart boundary and wrongly classifies heart as part of liver tissue as depicted in the Fig. 2-b. Figure 2-c shows the segmentation results with the proposed method. Figure 3 shows the overlay of segmented liver on its corresponding original image slice.

\section{CONCLUSION}

In this study, we have demonstrated a semiautomatic, robust and accurate method for segmentation of liver from CTA images for the purpose of extraction of anatomical, physiological and pathological information. The proposed method is robust to challenges hidden in liver segmentation namely intensity similarity of liver with neighboring organs, PVE. The success was mainly due to the new ARG method that combines intensity feature with the orientation of each pixel. The algorithm can be performed to segment organ structures like spleen, kidney for further analysis. As future work, data mining techniques can be employed to classify the patients with their liver anatomy.

\section{ACKNOWLEDGEMENT}

We would like to thank Senior Consultant-HPB and Liver Transplant, Dr Pradip Thomas Cherian of Global Health city, Hyderabad, India for the fruitful discussions and providing insight into the donor evaluation phase of Liver transplantation surgery. We also thank Dr Ramesh, Radiologist of Liberty Scans, Chennai, India for providing with the real datasets and support with manual segmentation during the preparation and implementation of this study.

\section{REFERENCES}

Ashraf, E., F. Coenen, M. Garcia-Finana and V. Sluming, 2012. Segmentation for medical image mining: A technical report. The University of Liverpool.

Badakhshannoory, H. and P. Saeedi, 2011. A modelbased validation scheme for organ segmentation in CT scan volumes. IEEE Trans. Biomed. Eng., 58: 2681-2693. DOI: 10.1109/TBME.2011.2161987 
Campadelli, P., E. Casiraghi and A. Esposito, 2009. Liver segmentation from computed tomography scans: A survey and a new algorithm. Artificial Intell. Med., 45: 185-196. DOI: 10.1016/j.artmed.2008.07.020

Chen, Y., Z. Wang, J. Hu, W. Zhao and Q. Wu, 2012. The domain knowledge based graph-cut model for liver CT segmentation. Biomed. Signal Proc. Control, 7 : 591-598. DOI: 10.1016/j.bspc.2012.04.005

Daisy, M.M.H. and S.T. Selvi, 2014. Soft computing based medical image retrieval using shape and texture features. Am. J. Applied Sci., 11: 258-265. DOI: 10.3844/ajassp.2014.258.265

Foruzan, A.H., R.A. Zoroof and M.Y.S. Hori, 2009. A Knowledge based technique for liver segmentation in CT data. Computerized Med. Imag. Graph., 33: 567-587. 10.1016/j.compmedimag.2009.03.008

Heimann, T. and H. Meinzer, 2009. Statistical shape models for 3D medical image segmentation: A review. Med. Image Anal., 13: 543-563. DOI: 10.1016/j.media.2009.05.004

Karthikeyan, S. and N. Rengarajan, 2014. Performance analysis of gray level co-occurrence matrix texture features for glaucoma diagnosis. Am. J. Applied Sci., 11: 248-257. DOI: 10.3844/ajassp.2014.248.257

Kumar, P.S. and P.G. Kumar, 2014. Performance analysis of brain tumor diagnosis based on soft computing techniques. Am. J. Applied Sci., 11: 329336. DOI: 10.3844/ajassp.2014.329.336
Lim, D.S.E., 2009. Automated segmentation of soft tissue in abdominal CT scans. MSc Thesis, School of Computing National University of Singapore.

Luo, S., J. Li and S. Seneviratne, 2012. Accurate nonrigid object segmentation in medical images by fusing statistical features with structural features. Patt. Recognit. Commun. Comput. Inform. Sci., 321: 404-412. DOI: 10.1007/978-3-642-33506$8 \_50$

Masoumi, A.B. and M.A. Pourmina, 2012. Automatic liver segmentation in MRI images using an iterative watershedalgorithm and Artifical neural networks. Biomed. Signal Proc. Control, 7: 429-437. DOI: 10.1016/j.bspc.2012.01.002

Xiang, S.Z., Y. Zhan, V.C. Raykar, G. Hermosillo and L. Bogoni et al., 2012. Mining anatomical, physiological and pathological information from medical images. ACM SIGKDD Explorat. Newslett., 14 : 25-34. DOI: $10.1145 / 2408736.2408741$

Yousuf, M.A. and M.R.H. Rakib, 2011. An effective image contrast enhancement method using global histogram equalization. J. Scientif. Res., 3: 43-50. DOI: $10.3329 /$ jsr.v3i1.5299

Zhang, X., J. Tian, K. Deng, Y. Wu and X. Li, 2010. Automatic liver segmentation using a statistical shape model with optimal surface detection. IEEE Trans. Biomed. Eng., 57: 2622-2626. DOI: 10.1109/TBME.2010.2056369 